\title{
Globe
}

Revue internationale d'études québécoises

\section{Pierre Pagé : Histoire de la radio au Québec, Montréal, Fides,} 2007

\section{Michel Filion}

Volume 11, numéro 2, 2008

URI : https://id.erudit.org/iderudit/1000535ar

DOI : https://doi.org/10.7202/1000535ar

Aller au sommaire du numéro

Éditeur(s)

Globe, Revue internationale d'études québécoises

ISSN

1481-5869 (imprimé)

1923-8231 (numérique)

Découvrir la revue

Citer ce compte rendu

Filion, M. (2008). Compte rendu de [Pierre Pagé : Histoire de la radio au Québec, Montréal, Fides, 2007]. Globe, 11(2), 228-230. https://doi.org/10.7202/1000535ar d'utilisation que vous pouvez consulter en ligne.

https://apropos.erudit.org/fr/usagers/politique-dutilisation/ 
de santé privées, et que près d'un Québécois sur deux est en faveur de la privatisation de la Société des Alcools du Québec (SAQ). Voilà des chiffres que l'Etat ne pourra ignorer lorsque viendra le temps d'élaborer des politiques dans ces domaines.

Cet ouvrage constitue donc un recueil de textes pertinent et accrocheur. Cependant, un dernier point mérite d'être souligné: le lecteur devra faire sa propre synthèse. En effet, dans sa conclusion, Robert Bernier ne fait que soulever les grandes lignes des dix-sept chapitres qui façonnent ce collectif. De quoi laisser le lecteur sur sa faim.

Magaly Brodeur École nationale d'administration publique

\section{Pierre Pagé \\ Histoire de la radio au Québec \\ Montréal, Fides, 2007.}

Le dernier livre de Pierre Pagé débute de manière engageante:

C'est une large perspective socioculturelle qui nous a guidé dans la préparation de ce volume sur l'histoire de la radio au Québec. Notre conviction profonde est qu'il faut voir ce média dans son époque comme un lien organique avec la société et toutes ses composantes institutionnelles, comme l'ont compris clairement les pionniers[]

peut-on lire en avant-propos. Pierre Pagé est bien connu dans le milieu pour avoir consacré plusieurs années de recherche à l'histoire de la radio. Il a grandement contribué à hisser ce média, longtemps négligé par les intellectuels, à la place qui lui revient dans notre cheminement collectif.

C'est donc avec beaucoup d'attentes qu'on entreprend la lecture de l'Histoire de la radio au Québec. Le titre est évocateur: enfin, un grand panorama qui permettra de comprendre comment et pourquoi la radio s'est développée au Québec de manière aussi distincte à travers le temps. Le lecteur est tourefois rapidement avisé: avec beaucoup de lucidité, l'auteur lui fait part des difficultés de reconstruire une telle histoire puisque les archives sont fragmentaires et parfois mal conservées. Les historiens de la radio connaissent bien cette situation. Les entreprises et les institutions radiophoniques, privées ou publiques, n'ont pas toujours su conserver leur patrimoine archivistique en jetant à la cave, quant ils n'étaient pas carrément détruits, des 
documents jugés révolus. Il faudra donc se résigner à une histoire partielle de la radio au Québec, ce qui n'est pas vraiment pour surprendre. «Notre recherche, malgré son envergure, n’a pas exploré tous les chemins de cet immense environnement sonore que la radio a créé pendant des décennies", précise l'auteur, qui ajoute: «Notre premier regret est certainement d'avoir été obligé par le réalisme de concentrer nos efforts sur la région de Montréal» (p. 9). Il est donc inexact de parler de l'histoire de la radio au Québec. Montréal demeure le centre de l'univers, une réalité dans le contexte québécois, mais une réalité tout de même réductrice pour un média de proximité. Même affiliées, les stations régionales du réseau de Radio-Canada ont produit une grande part de leur programmation, tout comme les stations privéés, et il faudra bien qu'un jour on s'y intéresse. Pierre Pagé dit d'ailleurs que «durant un demi-siècle, la radio s'est développée au Québec, dans toutes les régions, comme un instrument de cohésion sociale et d'ouverture sur le monde" (p. 17). Nous ne pouvons que lui donner raison et souhaiter que des recherches "en région" - selon l'expression désormais consacrée - viennent étoffer cette affirmation.

Cela dit, l'Histoire de la radio au Québec a de grands mérites. Elle a l'avantage de très bien situer de grands acteurs de la radio, comme le pionnier Jacques-Narcisse Cartier, pour ne citer que lui, de préciser avec force détails les dates et les circonstances de la création des premières stations, de décrire des moments forts comme la grève d'Asbestos, de définir l'éclosion de professions comme celle d'animateur, ou encore de cerner des genres radiophoniques, tels que le radiothéâtre, dont on ne dira jamais assez l'importance. Il s'agit d'une œuvre riche, incontournable même, bien qu'essentiellement narrative et événementielle, qui relève davantage des études littéraires que de l'histoire au sens global où on l'entend maintenant. Car, si elle permet de bien comprendre la culture de la radio depuis son émergence au Québec - il s'agit d'une contribution majeure -, elle le fait sans grand lien avec le contexte politico-économique canadien, pourtant déterminant à maints égards de l'évolution structurelle du système de radiodiffusion, et même de son contenu. Pour une perspective globale de l'histoire de la radio au Québec, il faudra lire cet ouvrage en parallèle avec d'autres travaux.

Aussi intéressant soit-il, il manque à ce livre un fil conducteur, une argumentation continue. La trentaine de chapitres, parmi lesquels certains ne font que trois pages, sont présentés sans suite logique, comme s'il s'agissait d'un assemblage d'articles et de travaux réalisés à plusieurs époques. D'ailleurs, le texte fait encore référence aux "Archives publiques du Canada», une appellation disparue depuis au moins vingt ans. Davantage qu'une grande 
synthèse historique, il faut voir dans ce livre différents portraits regroupés en cinq parties. La première parle de la genèse du média, la deuxième du journalisme et de l'information, la troisième de la fonction éducative et culturelle au service de la démocratie, la quatrième de l'art du son, c'est-à-dire de la musique, et la dernière partie est consacrée au théâtre radiophonique. On reconnaît là des sujets qui ont été chers à l'auteur tout au long de sa carrière. Enfin, la conclusion arrive abruptement. Pour l'importance du sujet et pour le haut niveau d'érudition de son auteur, ce livre aurait mérité un peu plus de soin. Présentée dans le désordre alphabétique, la bibliographie est incomplète et contient des redites. À l'occasion, on trouve aussi dans le texte des passages repris de manière quasi identique d'un chapitre à l'autre.

Malgré ces réserves et un titre quelque peu trompeur, l'Histoire de la radio au Québec enrichit notre connaissance par la somme des informations très précises qu'il contient, notamment dans sa chronologie descriptive. Cette publication permet certainement de mesurer toute la contribution de l'auteur à ce sujet au cours de sa carrière.

Michel Filion

Université du Québec en Outaouais 